\title{
Expression of E-cadherin and involucrin in leukoplakia and oral cancer: an immunocytochemical and immunohistochemical study
}

\author{
Alessandra Dutra da SILVA(a) \\ Bruna Jalfim MARASCHIN(b) \\ Natalia Koerich LAUREANO(b) \\ Natália DAROIT ${ }^{(b)}$ \\ Fernanda BROCHIER ${ }^{(b)}$ \\ Leonardo BÜNDRICH(b) \\ Fernanda VISIOLI(b) \\ Pantelis Varvaki RADOS(b) \\ (a) Universidade Luterana do Brasil, Department \\ of Oral Pathology, Canoas, RS Brazil. \\ (b) Universidade Federal do Rio Grande \\ do Sul - UFRGS, Department of Oral \\ Pathology, Porto Alegre, RS, Brazil.
}

Declaration of Interests: The authors certify that they have no commercial or associative interest that represents a conflict of interest in connection with the manuscript.

Corresponding Author:

Alessandra Dutra da Silva

E-mail: dra.alessandradutra@gmail.com

Submitted: Aug 22, 2016

Accepted for publication: Jan 24, 2017

Last revision: Feb 02, 2017
Abstract: To assess the immunocytochemical and immunohistochemical correlation of adhesion (E-cadherin) and cell differentiation (involucrin) molecules in oral leukoplakia and oral squamous cell carcinoma. Cytological samples and biopsies were obtained from male and female patients aged over 30 years with oral leukoplakia $(n=30)$ and oral squamous cell carcinoma $(n=22)$. Cell scrapings and the biopsy were performed at the site of the lesion and histological slides were prepared for the immunocytochemical analysis of exfoliated oral mucosal cells and for the immunohistochemical analysis of biopsy tissues using E-cadherin and involucrin. Spearman's correlation and kappa coefficients were used to assess the correlation and level of agreement between the techniques. Immunostaining for E-cadherin and involucrin by both techniques was similar in the superficial layers of the histological sections compared with cell scrapings. However, there was no statistical correlation and agreement regarding the immunocytochemical and immunohistochemical expression of E-cadherin and involucrin in oral leukoplakia $(\mathrm{R}=0.01, \mathrm{p}=0.958)($ Kappa $=0.017, \mathrm{p}=0.92)$ or in oral squamous cell carcinoma $(R=0.26, p=0.206)($ Kappa $=0.36, p=0.07)$. The immunoexpression of E-cadherin and involucrin in tissues is consistent with the expression patterns observed in exfoliated oral mucosal cells, despite the lack of a statistically significant correlation. There is an association of the histopathological characteristics of leukoplakia with the expression E-cadherin and of the microscopic aspects of oral squamous cell carcinoma with immunohistochemical expression of involucrin.

Keywords: Immunohistochemistry; Pathology; Mouth Neoplasms.

\section{Introduction}

Oral cancer is a public health problem. According to the National Cancer Institute, 11,140 new cases in men and 4,350 new cases in women have been estimated for Brazil for 2016. Survival and mortality rates have remained high for the past 30 years. ${ }^{1}$ Therefore, identification by biomarkers is necessary for the prevention of this disease in the general population. In several cases, cancer may be preceded by potentially malignant lesions, among which those the leukoplakia is highly prevalent and, therefore, of 
great clinical relevance. Even though it is not possible to predict the risk of malignancy of each leukoplakia, the presence of such lesions in the oral mucosa is a warning sign for patients and health professionals. ${ }^{2}$

Noninvasive methods, such as cytopathology, have proved effective in the early detection of cell changes that are not clinically visible in patients exposed to risk factors and in potentially malignant lesions. . $^{3,4,5,6}$ However, to have a better understanding of the risk of malignancy of each lesion, the correlation of cytopathology with complementary techniques such as immunocytochemistry may improve the effectiveness of this screening process. The expression of markers associated with cell adhesion (e.g., E-cadherin) and with cell differentiation (e.g., involucrin) may help predict the risk of malignancy, as their functions are dysregulated during carcinogenesis. ${ }^{78}$ E-cadherin is a glycoprotein that mediates cell-cell adhesion and that has been investigated in oral carcinogenesis. Some studies have indicated a reduction in the immunohistochemical expression of this molecule in early stages and during the progression of oral cancer. ${ }^{7,8}$

Proteins involved in the terminal differentiation of keratinocytes, such as involucrin, have also been found to have a low expression in biopsy tissues in leukoplakia associated with epithelial dysplasia when compared with oral mucosal cells exposed or not to carcinogens. ${ }^{9}$

The immunoexpression of these cell adhesion and differentiation markers has been investigated in different stages of carcinogenesis in biopsy tissues, but there are no studies on the expression of these proteins using cytopathological analysis of the oral mucosa. Hence, the aim of this study was to assess whether there exists a correlation between the immunocytochemical and immunohistochemical expression of E-cadherin and involucrin in oral mucosal cells of patients with oral leukoplakia and oral squamous cell carcinoma.

\section{Methodology}

\section{Sample selection}

The patients included in this study were recruited from the Outpatient Clinics and Centers for Dental
Specialties affiliated with the School of Dentistry of Universidade Federal do Rio Grande do Sul (UFRGS) and from the Division of Stomatology of Hospital Clinicas de Porto Alegre (HCPA), southern Brazil, between 2012 and 2014. The research was ethically conducted according to the Declaration of Helsinki. All patients signed a written informed consent form and the study protocol was approved by the Research Ethics Committee of UFRGS (protocol no. 12691813.6.0000.5347).

The sample included male and female patients aged over 30 years distributed into two groups: leukoplakia group $(n=30)$, with patients clinically diagnosed with oral leukoplakia, later confirmed by histopathological analysis; and the squamous cell carcinoma group $(n=22)$, with patients clinically diagnosed with primary oral squamous cell carcinoma confirmed by histopathological analysis. The following exclusion criterion was used: presence of other clinically visible oral lesions, except for squamous cell carcinoma and leukoplakia.

\section{Sample collection}

Cells from the oral mucosa at the site of the leukoplakia or squamous cell carcinoma were collected with a cytological brush (Cytobrush $\left.{ }^{\circledR}\right)$. The cytological smear was transferred onto a glass slide and fixed in an ethanol spray solution. Prior to the cell scrapings, all patients were asked to take off their removable prostheses and to rinse their mouths with filtered water for $1 \mathrm{~min}$.

After cell collection, the lesions (leukoplakia and squamous cell carcinoma) were biopsied. The collected material was fixed in $10 \%$ neutral buffered formalin and stained with hematoxylin and eosin at the Laboratory of Histopathology of the School of Dentistry at UFRGS for confirmation of the histopathological diagnosis.

Oral leukoplakias were subdivided into groups according to the absence or presence of dysplastic signs. The criteria used for the diagnosis of dysplasia were based on World Health Organization (WHO). Twelve architectural and cytological changes were investigated in the epithelial tissue and a minimum of four changes should be detected. 


\section{Immunocytochemical and Immunohistochemical technique}

Both immunocytochemical and immunohistochemical samples were submitted to the same protocol described by Silva et al. ${ }^{10}$ The oral smears were fixed in absolute alcohol solution and the histological sections of the paraffin blocks containing the biopsy specimens were previously deparaffinized in xylol. Both samples were processed for antigen retrieval, being immersed in a water bath at $95^{\circ} \mathrm{C}$ for $30 \mathrm{~min}$. Endogenous peroxidase was blocked by incubation in 3\% hydrogen peroxide. After washing, the sections were incubated with primary antibodies for E-cadherin (clone SY5, Abcam, Cambridge, UK) for $1 \mathrm{~h}$ and involucrin (clone 24E, cell signaling, Boston, USA), overnight. Peroxidase-linked secondary antibodies and chromogen incubation in diaminobenzidine tetrahydrochloride for $10 \mathrm{~s}$ for involucrin and for $5 \mathrm{~min}$ for E-cadherin (DAB; Envision HRP Kit, Dako Corp., Carpinteria, USA) were used to detect specific binding. The sections were counterstained with Harris hematoxylin, dehydrated, and mounted. A skin sample was used as positive control for both antibodies.

\section{Data analysis}

The slides were examined under a binocular light microscope coupled to a digital camera at 400X magnification. In the case of E-cadherin, positive cells were those whose membrane was stained whereas for involucrin, positive cells were those whose cytoplasm was stained. The immunocytochemical and immunohistochemical analyses of both molecules were semiquantitative and followed the criteria proposed by Lima et al. ${ }^{11}$ The following scores were used: $0=$ no positive cells, $1=$ up to $25 \%$ of positive cells, 2 up to $50 \%$ of positive cells, 3 up to $75 \%$ of positive cells, and $4=100 \%$ of positive cells. A semiquantitative analysis was also used to assess the agreement between the techniques, with the following scores: 0 no staining and $1=$ positive staining.

The slides were analyzed by a single, calibrated, and blinded examiner. The intra-examiner agreement was estimated by the intraclass correlation test $(\geq 0.75)$.

\section{Statistical analysis}

The data were submitted to the SPSS software (Statistical Package for the Social Sciences) for the statistical analysis. Spearman's correlation test was used to determine the association between the immunocytochemical and immunohistochemical findings obtained for E-cadherin and involucrin. The kappa coefficient was used to assess the agreement of immunocytochemical and immunohistochemical findings. Statistical significance was set at $5 \%(p<0.05)$.

\section{Results}

A total of 52 patients participated in the study (30 from the oral leukoplakia group and 22 from the oral squamous cell carcinoma group). Mean age was 57 years, with a range of 30 to 79 years. In the leukoplakia group, most patients were female while the opposite was observed in the squamous cell carcinoma group. Demographic and behavioral data are shown in Table 1.

Oral leukoplakia was subdivided into groups according to the histological specimens: dysplastic leukoplakia $(\mathrm{n}=7)$ and non-dysplastic leukoplakia

Table 1. Demographic and behavioral data about the patient population.

\begin{tabular}{lcc}
\hline \multirow{2}{*}{ Variables } & Leukoplakia group & Carcinoma group \\
\cline { 2 - 3 } Age (mean + SD) & $57( \pm 13.5)$ & $(\mathrm{n}=22)$ \\
Sex & & $57( \pm 8.4)$ \\
$\quad$ Female & $58.1 \%$ & $48.1 \%$ \\
$\quad$ Male & $41.9 \%$ & $51.9 \%$ \\
Smoking & $64.5 \%$ & $70.4 \%$ \\
Paci years (mean + SD) & $27( \pm 19.1)$ & $51( \pm 42.6)$ \\
Alcoholic beverage intake & $41.9 \%$ & $77.8 \%$ \\
Dental health & & \\
$\quad$ Poor & $54.8 \%$ & $63.6 \%$ \\
Regular & $16.1 \%$ & $22.7 \%$ \\
Good & $29 \%$ & $13.7 \%$ \\
Lesion site & & \\
Tongue & $32.2 \%$ & $18,10 \%$ \\
Floor of the mouth & $19.3 \%$ & $27.2 \%$ \\
Palate & $19.3 \%$ & $18.1 \%$ \\
Buccal mucosa & $12.9 \%$ & $9.1 \%$ \\
Bottom of Sulcus & $3.2 \%$ & - \\
Gingiva & $3.2 \%$ & - \\
\hline
\end{tabular}




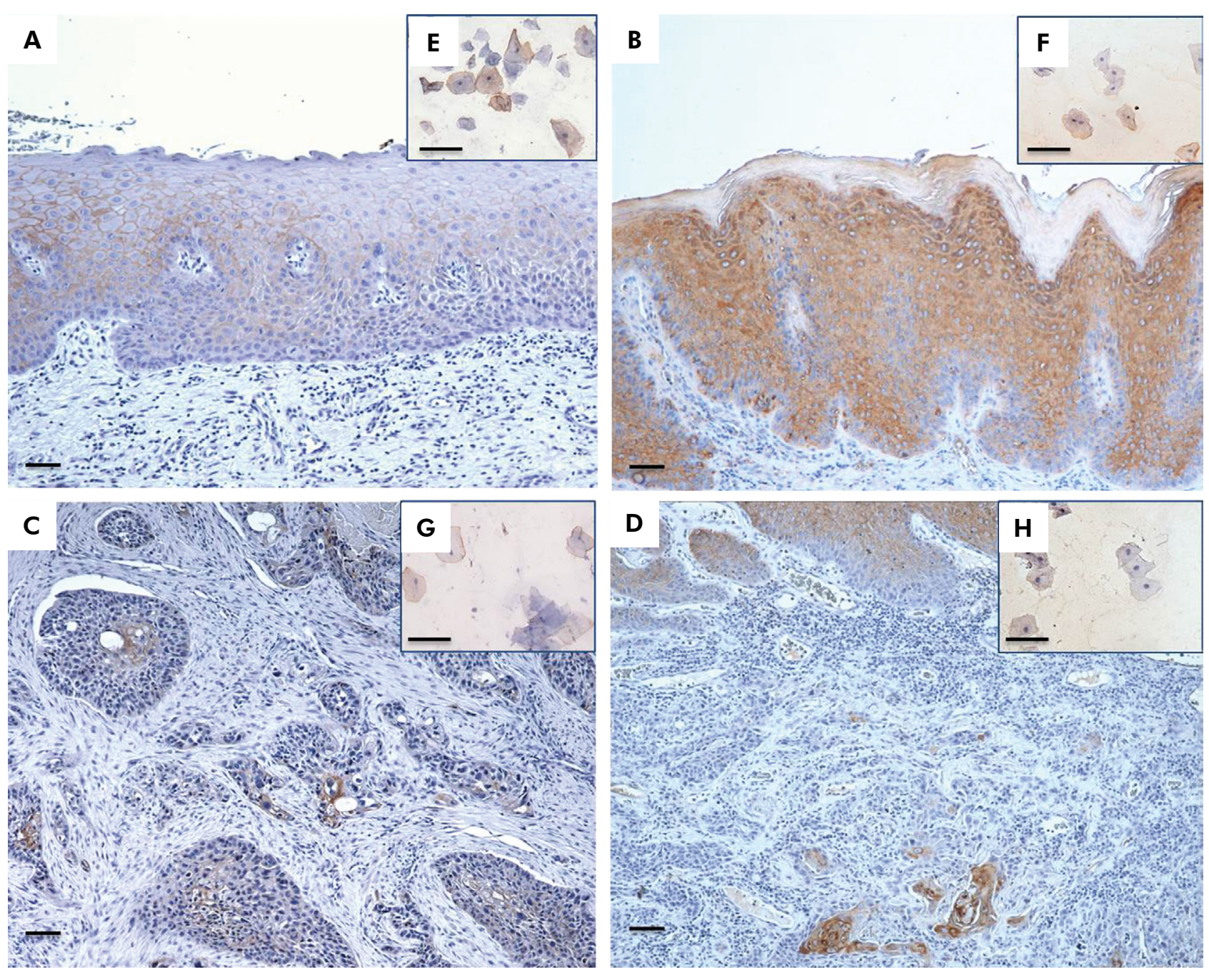

Figure 1. (A) Immunohistochemical expression of E-cadherin in the leukoplakia group, showing epithelial hyperplasia and acanthosis, and poor E-cadherin staining (100X). (E) In the upper right image, the cytologic smear indicates poor staining of E-cadherin in the leukoplakia group (400X). (C) Immunohistochemical expression of E-cadherin in the squamous cell carcinoma group, showing poor and focal staining at the center of tumor islands (100X). (G) In the upper right image, the cytologic smear indicates poor staining of $\mathrm{E}$-cadherin in the squamous cell carcinoma $\backslash$ group (400X). (B) Expression of involucrin in the leukoplakia group, with epithelial hyperplasia and hyperkeratosis, intense staining along the epithelium, except in the basal and suprabasal layers (100X). (F) In the upper right image, the cytologic smear indicates poor staining of involucrin in the leukoplakia group (400X). (D) Immunohistochemical expression of involucrin in the squamous cell carcinoma group, with focal staining only at the center of tumor islands, with keratin formation (100X). (H) In the upper right image, the cytologic smear indicates poor staining of involucrin in the squamous cell carcinoma group (400X).

( $n=23$ ). Most of non-dysplastic leukoplakias were histopathologically diagnosed as hyperkeratosis $(\mathrm{n}=8)$, epithelial hyperplasia and hyperkeratosis $(\mathrm{n}=11)$, and hyperkeratosis and acanthosis $(\mathrm{n}=4)$.

The immunohistochemical analysis revealed that the immunostaining of the superficial oral mucosal layers exhibited the same expression pattern as that of exfoliated cells, yielding weakly positive results for both groups, as shown in Figure 1.
The graphs presented in Figure 2 show the scores for the immunocytochemical and immunohistochemical analyses of E-cadherin and involucrin in the leukoplakia and squamous cell carcinoma groups.

Tables 2 and 3 display the results for the correlation between the immunocytochemical and immunohistochemical techniques in the leukoplakia and squamous cell carcinoma groups, indicating nonstatistically significant agreement. 
Moreover, there was no correlation of E-cadherin expression between the techniques in the leukoplakia $(\mathrm{R}=0.01, \mathrm{p}=0.958)$ and squamous cell carcinoma $(\mathrm{R}=0.26, \mathrm{p}=0.206)$ groups. Spearman's correlation of
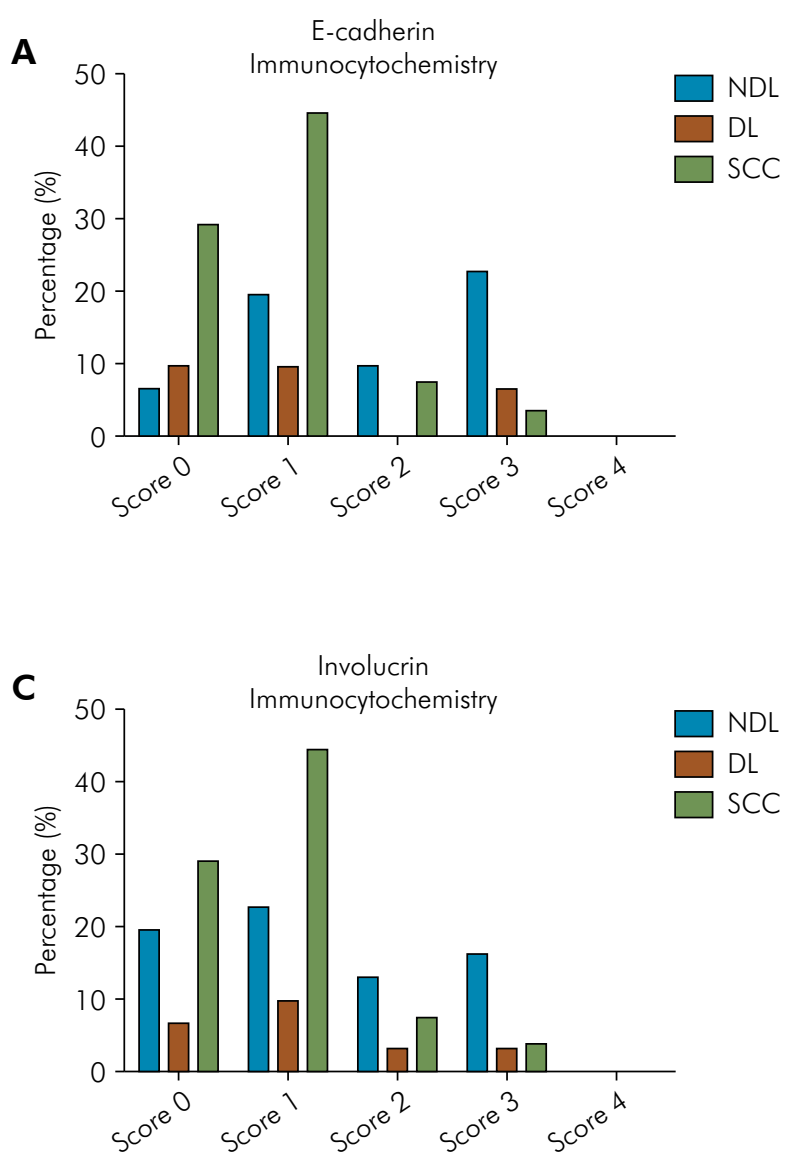

the immunocytochemical and immunohistochemical expression of involucrin was not statistically significant in the leukoplakia $(\mathrm{R}=0.15, \mathrm{p}=0.440)$ and squamous cell carcinoma $(R=0.15, p=0.532)$ groups.
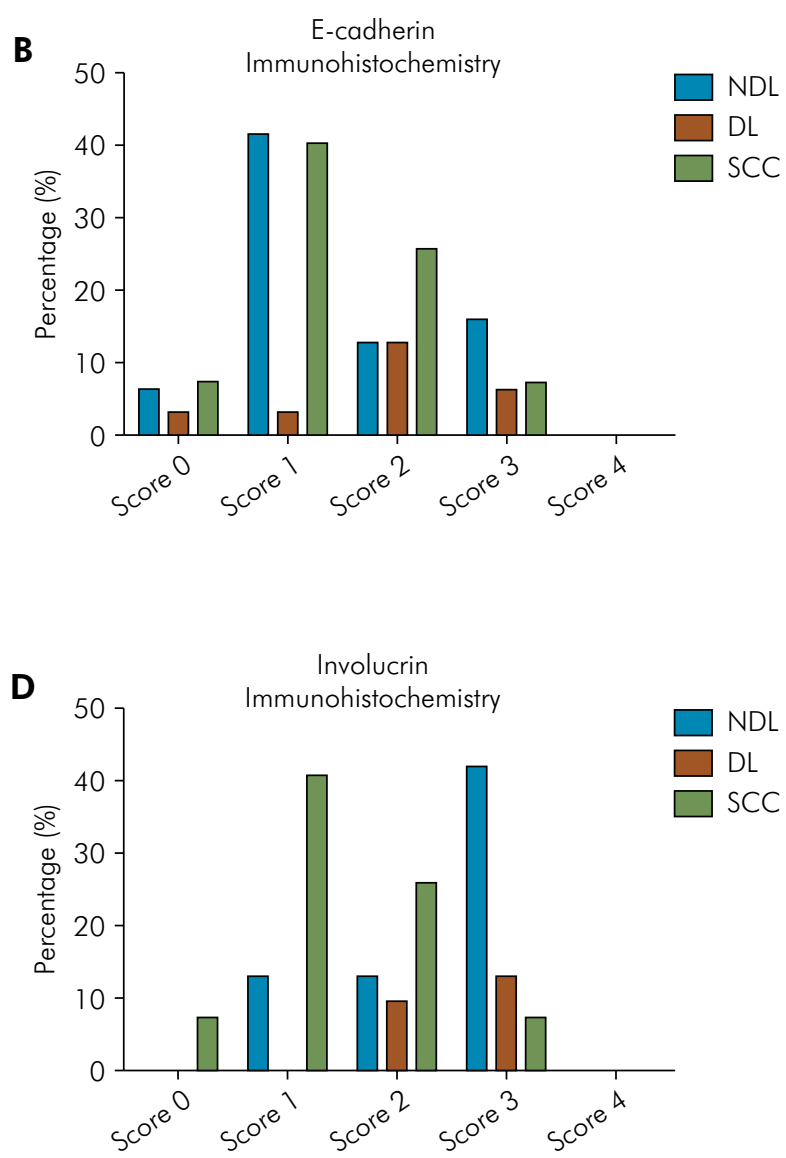

NDL: non-dysplastic leukoplakia group

DL: dysplastic leukoplakia group

SCC: squamous cell carcinoma group

Figure 2. Graphs comparing the scores of the immunocytochemical and immunohistochemical analyses of E-cadherin and involucrin in the leukoplakia and squamous cell carcinoma groups.

Table 2. Level of agreement between immunocytochemical and immunohistochemical expression of E-cadherin in the leukoplakia and squamous cell carcinoma groups.

\begin{tabular}{|c|c|c|c|c|}
\hline \multirow{2}{*}{ Variable } & \multicolumn{4}{|c|}{ Immunocytochemistry E-cadherin } \\
\hline & Score 0 & Score 1 & Total & Kappa \\
\hline \multicolumn{5}{|c|}{ Leukoplakia Group } \\
\hline Score 0 & $10(58.8 \%)$ & $6(41.2 \%)$ & $16(100 \%)$ & 0.017 \\
\hline Score 1 & $8(57.1 \%)$ & $6(42.9 \%)$ & $14(100 \%)$ & - \\
\hline \multicolumn{5}{|c|}{ Squamous Cell - Carcinoma Group } \\
\hline Score 0 & $10(75 \%)$ & $4(25 \%)$ & $14(100 \%)$ & 0.36 \\
\hline Score 1 & $3(37.5 \%)$ & $5(62.5 \%)$ & $8(100 \%)$ & - \\
\hline
\end{tabular}


Table 3. Level of agreement between immunocytochemical and immunohistochemical expression of involucrin in the leukoplakia and oral squamous cell carcinoma groups.

\begin{tabular}{ccccc}
\hline \multirow{2}{*}{ Variable } & \multicolumn{4}{c}{ Immunocytochemistry E-cadherin } \\
\cline { 2 - 5 } & Score 0 & Score 1 & Total & Kappa \\
\hline Leukoplakia Group & & & \\
Score 0 & $2(40 \%)$ & $3(60 \%)$ & $5(100 \%)$ & 0.12 \\
Score 1 & $2(40 \%)$ & $3(60 \%)$ & $5(100 \%)$ & - \\
Squamous Cell - Carcinoma Group & & \\
Score 0 & $11(91.7 \%)$ & $1(8.3 \%)$ & $12(100 \%)$ & 0.07 \\
Score 1 & $6(85.7 \%)$ & $1(14.3 \%)$ & $7(100 \%)$ & - \\
\hline
\end{tabular}

\section{Discussion}

With the advances in cancer research in recent years, screening, diagnostic, and prognostic methods have evolved substantially. New techniques, such as immunohistochemistry, were incorporated into histopathological analyses. The use of this technique is well established in the literature for the investigation of different tissue proteins. ${ }^{12}$ This is the first study to assess the expression of adhesion (E-cadherin) and cell differentiation (involucrin) markers in exfoliated oral mucosal cells from leukoplakia and squamous cell carcinoma lesions using immunocytochemistry. In addition, the findings of the immunocytochemical and immunohistochemical analyses were correlated by using these markers in patients with the lesions described above.

The demographic aspects of our sample showed a predilection for females in the leukoplakia group. This finding is in disagreement with those of other authors, who reported a higher prevalence among men, though sex distribution may vary according to the characteristics and population habits associated with risk factors. ${ }^{13}$ In addition, individuals in the leukoplakia group had a higher consumption of tobacco than of alcohol, so we consider tobacco to be the main etiologic factor of leukoplakias in our sample, as previously described by other authors. ${ }^{13,14}$ Most individuals presented poor oral hygiene in both groups. The most common sites of lesions were the tongue and floor of the mouth, which are the locations mostly affected by oral squamous cell carcinoma. ${ }^{13}$

Many authors have suggested that low E-cadherin immunoexpression could indicate tumor progression in leukoplakia. ${ }^{78,15,16}$ This finding is in line with Santos-García et al., ${ }^{15}$ in which $20 \%$ of leukoplakias with epithelial dysplasia and $73 \%$ of carcinomas in situ and microinvasive carcinoma had poor E-cadherin expression. Our results corroborate those of previous studies, since we observed that the expression of E-cadherin is in agreement with the severity of epithelial changes observed in leukoplakia and dysplastic oral leukoplakia showed lower immunocytochemical expression of this molecule than non-dysplastic leukoplakia. This suggests that the immunocytochemical staining of E-cadherin can signal dysplastic events in leukoplakia.

The immunohistochemical expression of E-cadherin in the leukoplakia and squamous cell carcinoma groups revealed higher staining of this molecule in the intermediate and suprabasal layers of epithelial tissue and poor or no staining in more superficial layers, as observed in cell scrapings, which contained only superficial cells with poor immunostaining (Figure 1AC).

Regarding involucrin immunoexpression, most histological tissues expressed it in the most superficial layer (stratum corneum), as detected in oral mucosal smears (Figure 1FH). Involucrin is associated with terminal differentiation of epithelial cells, so it can display varying expression in leukoplakia, according to the degree of cell differentiation or cell atypia observed in each case. Involucrin expression in the leukoplakia group was observed in the whole epithelium, except in the superficial layer. However, other authors have found differences in involucrin expression in leukoplakias with higher severity of epithelial dysplasia. Dysplastic leukoplakia with mild to moderate dysplasias showed high involucrin expression (> 50\% of positive cells), compared with leukoplakia with severe dysplasia, which showed lower expression of this marker $(<25 \%$ of positive cells). ${ }^{17,18}$ In our work, the histological grading of epithelial dysplasia was not performed due to the small sample size and moderate involucrin expression was observed in all leukoplakic lesions.

Our findings for the oral squamous cell carcinoma group showed immunohistochemical staining of involucrin only in the cells at the center of tumor islands, characterized by well-differentiated cells 
with keratin pearls, as pointed out in other studies, which demonstrated that carcinoma in situ and invasive carcinoma had different involucrin expression patterns, which was closely related to the degree of differentiation of the tumors. ${ }^{17,19}$

The qualitative analysis of E-cadherin and involucrin immunoexpression in both techniques was similar and coherent in the superficial layers of the histological sections compared with the cell scrapings, although no statistically significant correlation could be established between the immunocytochemical and immunohistochemical techniques.

Our analysis of exfoliated oral mucosal cells demonstrates that E-cadherin and involucrin immunoexpression occurs only in cells from the superficial epithelial layers in the final stage of maturation. The immunocytochemical technique does not allow assessing cells from deeper layers wherein these proteins could have a positive expression. This differs from the findings obtained

\section{References}

1. National Cancer Institute José Alencar Gomes da Silva. [Estimative 2016: cancer incidence in Brazil]. Rio de Janeiro: Instituto Nacinal do Câncer; 2016[cited 2016 Jun 1]. Portuguese. Available from: http://www.inca.gov.br/estimativa/2016

2. Warnakulasuriya S, Johnson NW, Waal I. Nomenclature and classification of potentially malignant disorders of the oral mucosa. J Oral Pathol Med. 2007;36(10):575-80. https://doi.org/10.1111/j.1600-0714.2007.00582.x

3. Gonzalez Segura I, Secchi D, Carrica A, Barello R, Arbelo D, Burgos A et al. Exfoliative cytology as a tool for monitoring pre-malignant and malignant lesions based on combined stains and morphometry techniques. J Oral Pathol Med. 2015;44(3):178-84. https://doi.org/10.1111/jop.12219

4. Paiva RL, Sant'Ana Filho M, Bohrer PL, Lauxen IS, Rados PV. AgNOR quantification in cells of normal oral mucosa exposed to smoking and alcohol: a cytopathologic study. Anal Quant Cytol Histol. 2004;26(3):175-80.

5. Burzlaff JB, Bohrer PL, Paiva RL, Visioli F, Sant'Ana Filho M, Silva VD. Exposure to alcohol or tobacco affects the pattern of maturation in oral mucosal cells: a cytohistological study. Cytopathology. 2007;18(6):367-75. https://doi.org/10.1111/j.1365-2303.2007.00473.x

6. Acha A, Ruesga MT, Rodríguez MJ, Martínez de Pancorbo MA, Aguirre JM. Applications of the oral for histological tissues in which it was possible to detect immunohistochemical expression of these proteins in all epithelial tissue layers.

Accordingly, our findings indicate that there is an association of the histopathological characteristics of leukoplakia with E-cadherin expression and microscopic aspects of oral squamous cell carcinoma with immunohistochemical expression of involucrin. The correlation between the immunocytochemical and immunohistochemical expression of these proteins was not statistically significant in oral leukoplakia and oral squamous cell carcinomas; however, the qualitative analysis showed that the expression of these molecules in both techniques was coherent. Therefore, it is necessary to broaden the knowledge about the immunoexpression of other biomarkers expressed in superficial epithelial layers using immunocytochemistry in order to correlate it with their immunohistochemical expression in formalin-fixed and paraffin-embedded tissues. scraped (exfoliative) cytology in oral cancer and precancer. Med Oral Patol Oral Cir Bucal. 2005;10(2):95-102.

7. Yogesh TL, Narayan TV, Shreedhar B, Shashidara R, Leekymohanty. The expression of E-cadherin and cathepsin-D in normal oral mucosa, oral epithelial dysplasia and oral squamous cell carcinoma: a comparative analysis between immunohistochemistry and routine histopathology. J Oral Maxillofac Pathol. 2011;15(3):288-94. https://doi.org/10.4103/0973-029X.86689

8. Sridevi U, Jain A, Nagalaxmi V, Kumar UV, Goyal S. Expression of E-cadherin in normal oral mucosa, in oral precancerous lesions and in oral carcinomas. Eur J Dent. 2015;9(3):364-72. https://doi.org/10.4103/1305-7456.163238

9. Gilchrist JM, Thompson GC, Medina JE. Markers of keratinocyte differentiation in snuff-induced leukoplakia. Am J Surg. 1992;164(6):563-6. https://doi.org/10.1016/S0002-9610(05)80707-X

10. Silva AD, Lima CF, Maraschin BJ, Laureano NK, Daroit NB, Brochier $\mathrm{F}$ et al. Immunocytochemistry associated with oral exfoliative cytology: methodological analysis. Anal Quant Cytopathol Histopathol. 2015;37(2):134-8.

11. Lima CF, Crastechini E, Issa JS, Balducci I, Cabral LA, Almeida JD. Evaluation of apoptotic pathway in oral mucosa by smoking in a Brazilian Outpatient Smoking Cessation Program. Int J Cardiol. 2015;184:514-6. https://doi.org/10.1016/j.ijcard.2015.02.103 
12. Hardy LB, Fitzgibbons PL, Goldsmith JD, Eisen RN, Beasley MB, Souers RJ et al. Immunohistochemistry validation procedures and practices: a College of American Pathologists survey of 727 laboratories. Arch Pathol Lab Med. 2013;137(1):19-25. https://doi.org/10.5858/arpa.2011-0676-CP

13. Reichart PA. Identification of risk groups for oral precancer and cancer and preventive measures. Clin Oral Investig. 2001;5(4):207-213. https://doi.org/10.1007/s00784-001-0132-5

14. Amagasa T, Yamashiro M, Uzawa N. Oral premalignant lesions: from a clinical perspective. Int J Clin Oncol. 2011;16(1):5-14. https://doi.org/10.1007/s10147-010-0157-3

15. Santos-García A, Abad-Hernández MM, Fonseca-Sánchez E, Julián-González R, Galindo-Villardón P, Cruz-Hernández JJ et al. E-cadherin, laminin and collagen IV expression in the evolution from dysplasia to oral squamous cell carcinoma. Med Oral Patol Oral Cir Bucal. 2006;11(2):E100-5.
16. Zeidler SV, Botelho TL, Mendonça EF, Batista AC. E-cadherin as a potential biomarker of malignant transformation in oral leukoplakia: a retrospective cohort study. BMC Cancer. 2014;14(1):972. https://doi.org/10.1186/1471-2407-14-972

17. Vigneswaran N, Peters KP, Hornstein OP, Haneke E. Comparison of cytokeratin, filaggrin and involucrin profiles in oral leukoplakias and squamous

carcinomas. J Oral Pathol Med. 1989;18(7):377-90. https://doi.org/10.1111/j.1600-0714.1989.tb01569.x

18. Itoiz ME, Conti CJ, Gimenez IB, Lanfranchi HE, Fernandez-Alonso GI, Klein-Szanto AJ. Immunodetection of involucrin in lesions of oral mucosa. J Oral Pathol. 1986;15(4):205-8. https://doi.org/10.1111/j.1600-0714.1986.tb00608.x

19. Toto PD, Nadimi H. Coexpression of cytokeratins, involucrin, and blood group antigens in oral squamous cell carcinomas. Oral Surg Oral Med Oral Pathol. 1990;70(1):75-80. https://doi.org/10.1016/0030-4220(90)90182-R 